Rev. Elev. Méd. vêt. Pays trop., 1968, 21, 3 (293-96).

\title{
Note sur la peste bovine expérimentale du dromadaire
}

\author{
par A. PROVOST, Y. MAURICE ef C. BORREDON \\ (I. E. M. V. T., Laboratoire de Recherches Vétérinaires de Farcha, Fort-Lamy, Tchad)
}

\begin{abstract}
RÉSUMÉ
Un seul dromadaire du Tchad (Camelus dromaderius) sur dix ayant reçu un aérosol de virus bovipestique a répondu par une maladie asymptomatique (leucopénie, apparition d'anticorps antıpestiques neutralisant), non conłagleuse. Quelques facteurs pouvant gouverner cette réceptivité réduite sont discutés.
\end{abstract}

Dans une note précédente, un sondage sérologique a montré que 7,7 p. 100 des dromadaires vivant dans le sahel tchadien possédaient des anticorps antıbovipestiques décelables par les tests de séro-neutralisation et d'inhibition de l'hémagglutination morbilleuse (3). L'origine de ces anticorps spécifiques ne pouvant que se rapporter à une infection pestique clinique ou occulte, il devenait normal d'étudier la maladie expérimentale du dromadaire et de se demander quel rôle pouvait jouer cette espèce dans l'épizootiologie de la pesie bovine. La réponse à ces deux questions fait l'abjet de cette note.

\section{MATÉRIEL ET MÉTHODES}

1. Méthode d'infection. On donne la préférence à l'cérosol infectieux qui reproduit les modalités de la transmission naturelle du contage (6). Pour ce faire, on utilise un générateur d'aérosols médicamenteux Jouan délivrant des particules de l'ordre du micron et débitant avec son compresseur d'origıne $12 \mathrm{ml}$ de liquide à l'heure. Une exposition de cınq minutes des anımaux correspond donc à $1 \mathrm{ml}$ (soit on le verra, à $10^{5,3} \mathrm{DCP}_{50}$ de virus pestique). Pour l'insufflation, un manchon de matière plastique souple relie le générateur au mufle du chameau.
2. Souche de virus pestique. En l'absence de souche de virus de la peste bovine isolée de l'espèce cameline, c'est la souche DK de virus bovipestique qui a servi aux expériences. Cette souche d'origine sénégalaise est entretenue à Farcha par passages naturels de bœuf malade à bœuf réceptif à l'exclusion de l'inoculation. On s'efforce, par cet artifice, de préserver ses qualités de contagiosité immédiate afin de ne pas rencontrer les difficultés qui existent dans la manipulation des souches de laboratoire peu ou pas contagieuses, comme la souche Kabete $O$.

Le matériel virulent est un broyat de ganglions lymphatiques et de rate de bouf atieint clinıquement de peste, dilué au $1: 10$ en peptone d̀ 5.5 p. 100 en eau distıllée et lyophilisé. II est réhydraté avec de l'eau distillée glacée à son volume initial juste au moment de l'emploi et centrifugé pour sédımenter d'éventuels débris.

Le titrage en cultures cellulaires de rein d'embryon de veau lui assigne le titre de $10^{5,3}$ $\mathrm{DCP}_{50}$ par ml.

Son pouvoir pathogène est contrôlé par un aérosol administré à un zébu sensible en même temps qu'aux chameaux de l'expérience, zébu qui a contracté la peste et dont il ne sera plus fait mention dans le compte rendu. 
3. Animaux d'expérience. Dix dromadaires (Comelus dromaderius) entrent en expérience : sept sont originaires de la régıon d'Ati, trois de celle de Mao. Leur âge n'est pas précisé, maıs tous dix sont adultes. Ils sont logés en box individuels contigus, séparés par des murets de un mètre de haut et peuvent ainsi avoir des contacts entre eux. Une prise de sang est effectuée avant l'aérosol et les 10 ou $12^{\mathrm{e}}$ jours pour les 7 premiers anımaux, le $18 \mathrm{e}$ jour pour 2 des 3 derniers (le $3^{\mathrm{e}}$ de ce groupe est mort accidentellement 7 jours après |'aérosol).

Deux jours avant l'infection expérimentale puis tous les jours ensuite, on prend la température rectale avec des thermomètres séparés et on pratique une numération leucocytare (numération totale). Eventuellement, des frottis de sang sont effectués pour défecter des hémoprotozoaires.

Les autopsies sont pratiquées d̀ des temps variables : du 10 au 12e jour pour les 7 premiers chameaux, les 7 et 20 e jours pour les autres. On récolte les amygdales pour effectuer des coupes histologiques et les ganglıons mésentériques pour y rechercher le précipitogène bovipestique.

Le 3 jour après l'aérosol virulent, on place en contact de chaque chameau un zébu réceptif à la peste. La réceptivité de ces bovins est attestée i la fois par leur achat en République Centrafricaine, territoire non infecté de peste bovine et où de ce fait aucune vaccination antipestique n'est pratiquée, ef par l'absence d'anticorps neutralisant le virus pestique dans leurs sérums. Zébus et chameaux d'un box reçoivent le même affouragement et s'abreuvent au même point d'eau. Leur température est prise tous les jours avec un thermomètre différent. On n'a pas suivi leur formule leucocytaire. Une prise de sang est effectuée trois semaines après la mise en contact. Ils sont ensulte inclus dans une expérience d'immunité antipestique.

4. Tests sérologiques.

a) Séro-neutralisation en cultures cellulaires, suivant les modalités techniques de PLOWRIGHT ef FERRIS (5), sans inactivation thermique des sérums.

b) Inhibition de l'hémagglutınation morbilleuse, selon la méthodologie de BÖGEL, ENDERS RUCKLE et PROVOST (1). c) Précipitation-diffusion en gélose, pour la recherche de l'antıgène pestique d'après SCOTT et BROWN (10).

\section{RÉSULTATS}

\section{A. Concernant les dromadaires.}

1. Observations cliniques. Un seul dromadaire ( $\mathrm{n}^{\circ}$ 2576) a présenté un accès de fièvre qui a pu être rapportée à la présence de Tryponosoma evansi; traité, it retournait à la normale 48 heures plus tard. On a pu observer des trypanosomes lors des numérations leucocytaires chez deux autres chameaux ( $n^{\text {os }} 1$ ef 2 ), ceci sans hyperthermie,

Chez le chamequ no 1 , on a remarqué un peu de larmoiement qu'il est difficile de rapporter à la peste ou à la trypanosomiase concomitante bien supportée.

Trois chameaux (nos 1, 2 et 2575) ant manifesté le 5 e jour après l'aérosol une forte hyperleucocytose suivie d'une leucopénie. Pour deux d'entre eux (nos 1 et 2), le retour à la normale s'est fait en une semaine; le troisième animal étcit abattu pour les besoins d'une expérience menée simultanément à celle-ci.

2. Observations nécropsıques. Aucune lésion attribuable d̀ la peste n'a été remarquée d̀ l'autopsie. L'image histologique des amygdales était normale, sans plasmodes multinucléés. La recherche $d u$ précipitogène pestique a été chez tous négative.

3. Observotions sérologiques. Les épreuves de séro-neutralisation n'ont pu détecter d'anticorps antobivipestiques dans les sérums prélevés avant l'aérosol.

Des anticorps neutralisants au taux relativement modeste de $\mathrm{TN}_{\mathbf{5 0}}$ : $10^{0,9}$ sont apparus le $18 \mathrm{e}$ jour après l'infection dans le sérum d'un seul chameau; la réaction d'inhibition de l'hémagglutınation morbilleuse est restée négative.

\section{B. Concernant les zébus en contact.}

Aucun des zébus n'a présenté le moindre trouble. Leur sérologie pestique est demeurée négative. Inclus dans des épreuves d'immunité antiseptique, ils ont ensuite prouvé leur parfaite réceptivité au virus. 


\section{DISCUSSION}

Les conclusions de cette expérience sont aisées à tirer. Avec une contamination simulant au mieux les conditions naturelles ef un virus pestique d'origine bovine, un seul dromadaire sur dix contamınés fait une peste bovine asymptomatique, occulte, non contagieuse. La seule preuve de son infection réside dans sa conversion sérologique. Il est difficile d'affirmer que la leucopénie enregistrée sur deux chameaux soit authentiquement d'origine pestique puisque l'un des animaux n'a pas élaboré d'anticorps antipestiques 18 jours après l'aérosol. Quant aux autres on peut penser qu'ils n'ont pas fait la peste, ce qui semble être prouvé par la négatvité simultanée des images histologiques, de la recherche du précipitogène et de la sérologie : dans le cas de peste, on peut s'attendre à ce que l'une au moins des trois recherches soit concluante en l'absence de la positivité des deux autres.

Point remarquable, la maladie asymptomatique du dromadaire n'est pas contagieuse comme si, ce qui n'a pas été recherché, le virus n'était pas excrété. Peut-on voir dans cette absence de contagiosité, la faible montée des anticorps neutralisant et la négavité de la recherche des anticorps inhibant l'hémagglutination morbilleuse, une faiblesse de la replication du virus bovipestique chez l'espèce camelıne? C'est fort probable au regard de ce qu'enseigne le comportement du virus dons d'autres espèces connues comme peu réceptives, dont la chèvre, chez lesquelles existe une dissociation des anticorps. neutralisant et inhibant (7).

Jusqu'à plus ample informé, on ne peut incriminer le dromadare d'être un rela actif dans l'épizootiologie bovipestique ce qui, au demeurant, est en parfait accord avec les observations faites sur le terraın : le chameau n'a jamaıs été accusé au Tchad d'être à l'origine de foyers de peste non plus que de souffrir de la maladie.

On peut faire quelques réflexions sur cette apparente réceptivité réduite du dromadaire. Pourquoi, alors que neuf de ses congénères résistent au contage, un seul sujet s'ınfecte-t-il ? Il ne paraît pas spécialement valable d'accuser un affaiblıssement du terraın dû à la seule trypanosomiase, car deux autres chameaux _- au moins - chez lesquels on a noté la pré- sence de $T$. evonsı auraient alors do s'infecter eux aussi.

La maladie infectieuse se jove à deux partenaires : l'hôte et l'agent infectieux. Si dans le cas précent il semble bien que l'on puisse suspecter l'espèce cameline de n'avoir qu'une réceptivité réduite à la peste, ne peut-on penser aussi que la source de virus jove un rôle? La souche de virus qui a été utilisée est d'origine bovine, contınuellement passée sur bouf réceptif. Cette même souche DK se montre incapable de contamıner les chèvres (7). N'est-ce pas d'ailleurs ce qui se passe dans la nature : felle souche de virus pestique infecte telle espèce à l'exclusion de telle autre, comme si il y avait une spécialisation de certaınes souches. Le plus bel exemple en est donné par le virus de la peste des petits ruminants, mais le falt existe pour d'autres souches connues du virus pestique : la souche Vom isolée par JOHNSON en 1957 (2) se montrait capable d'infecter natureltement le mouton ef rendalt cette espèce vectrice du contage pour le bœuf; la souche Ol Balbal de ROBSON et al. (8) a un pouvoir pathogène patent pour le seul élan de Derby et les souches de PLOWRIGHT (4), isolées d'artiodactyles sauvages, n'ont qu'une virulence des plus réduites pour le bouf.

D'après les résultats des sondages sérologiques effectués sur des dromadaires vivants en zone d'enzootie pestıque et ceux de la présente expérience, il semble bien que le chameau ne soit que peu réceptif à la peste. II ne paraît toutefois pas illogique de prétendre que des mutants pulssent se faire jour pour atteindre le chameau comme cela est rapporté dans la littérature (3). Au passage, on ne peut s'empêcher de penser que l'enquête négative de SCOTT et MACDONALD (11) a pu être faussée à la fois par le trop petit nombre de sérums de chameaux examinés (30 sérums de dromadaires ayant vécu dans un foyer de peste), mais aussi parce que la souche de virus avec laquelle ces animaux avalent été en contact paraissaıt être «spécialisée» pour les artiodactyles sauvages.

Nous avons pris connassance des résultats expérimentaux de SINGH et ATA (9) alors que les essais d'infection ici relatés venaient d'être achevés ; il est intéressant d'y constater que le virus-vaccin de cultures cellulaires semble se multiplier très mal chez le chameau ainsi qu'en 
atteste la faible conversion sérologique $(1: 2)$ de deux chameaux sur quatre, les deux autres étant restés négatifs; nous aimons à y voir une adaptation bovine de la souche vaccinale ayant des difficultés d̀ se repliquer dans des cellules camelines, argument qui s'ajoute aux vues ci-dessus exposées. Comme le soulignent SINGH et ATA, et ce sera là aussi notre conclusion, l'espèce cameline est apparemment peu réceptive au virus pestique; on peut ajouter que cela peut ne pas toujours être vrai si un mutant adapté à l'espèce apparaissait.

\section{RÉFÉRENCES}

1. BÖGEL (K.), ENDERS-RUCKLE (G.) et PROVOST $(A$.$) . - Une réaction sérologique$ rapide de mesure des anticorps antibovipestiques. C. R. Acod. Sci. (Poris), 1964, 259, 482-484.

2. JOHNSON (R. H.). - An outbreak of rinderpest involving cattle and sheep. Vet. Rec., 1958, 70, 457-461.

3. MAURICE (W.), PROVOST (A.) et BORREDON (C.). - Présence d'anticorps antibovipestiques chez le dromadaire du Tchad. Rev. Elev. Méd. vét. Pays trop., 1967, 20, 537-542.

4. PLOWRIGHT (W.). - Some properties of strains of rinderpest virus recently isolated. in E. Africa. Res. Vet. Scl., 1963, 4, 96-108.
5. PLOWRIGHT (W.) et FERRIS (R. D.). Studies on rinderpest virus in tissue culture. III : The stability of cultured virus and its use in neutralization tests. Arch.ges. Virusfors, $1961,11,516-533$.

6. PROVOST (A.). - Essais de transmission de la peste bovine par aérosols virulents. Bull. epizoot. Dis. Afr. 1958, 6, 79-85.

7. PROVOST (A.), BORREDON (C.), MAURICE (Y.) et QUEVAL (R.). - Epizootiologie de la peste bovine. In ": Rapport annuel du Laboratoire de Recherches vétérinaires et zootechniques de Farcha, 1967, tome 1.

8. ROBSON (J.), ARNOLD (R. N.), PLOWRIGHT (W.) et SCOTT (G.R.). - The isolation from an eland of a strain of rinderpest virus attenuated for cattle. Bull. epizoot. Dis. Afr., 1959, 7, 97-102.

9. SINGH (K. V.) et ATA (F.). - Experimental rinderpest in camels. A preliminary report. Bull. epizoot. Dis. Afr., 1967, 15, 19-23.

10. SCOTT (G. R.) et BROWN (R.D.).-- Rinderpest diagnosis with special reference to the agar gel double diffusion test. Bull. epizoot. Dis. Afr. 1961, 9, 83-120.

11. SCOTT (G. R.) et MACDONALD (J.). Kenya camels and rinderpest. Bull. epizoot. Dis. Afr., 1962, 10, 495-497.

\section{SUMMARY}

A note on experimental rinderpest in the dromedary

Only one out of ten Chad dromedaries (Comelus dramaderius) infected with rinderpest virus by aerosol, showed evidence of asymptomatic, non-contagious Infection (leucopaenı, appearance of neutralising antibodies of rinderpest). Some of the factors which could govern this reduced susceptibility are discussed.

\section{RESUMEN}

Nota sobre la pesfe bovina experimental del dromedario

Se encontró una enfermedad asintomática (leucopenia, aparición de anticuerpos antipesticos neutralizantes), no contagiosa, en un solo dromedario del, Chad (Camelus dromaderius) de diez después de la administración de un aerosol de virus bovipestico. Se discutan algunos factores pudiendo determinar ésia receptividad. 